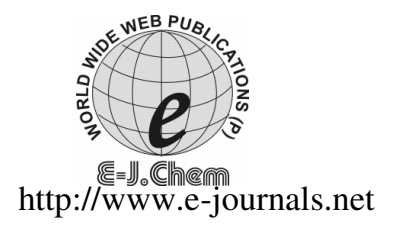

ISSN: 0973-4945; CODEN ECJHAO

E-Journal of Chemistry

2010, 7(1), 234-238

\title{
Synthesis of Some Phenylpyrazolo Benzimidazolo Quinoxaline Derivatives as Potent Antihistaminic Agents
}

\author{
C. H. SRIDEVI*, K. BALAJI, A. NAIDU and R. SUDHAKARAN \\ Dept. of Pharmaceutical Chemistry, Geethanjali College of Pharmacy, \\ Cheeryal(V),Keesara(M), Hyderabad-501301, India. \\ sridevi.phd@gmail.com
}

Received 22 May 2009; Accepted 15 July 2009

\begin{abstract}
Diphenyl quinoxaline (NI) was fused with benzimidazole (NII) by a methylene bridge, which was then allowed for acetylation. The acetylated product (NIV) was made to react with different aromatic aldehydes to give chalcones (NV1-NV5). Chalcones refluxed with substituted acid hydrazides to afford different phenyl pyrazolo benzimidazole quinoxaline derivatives (NVI 1-NVI 15). The structure of chalcones and phenyl pyrazolo benzimidazole quinoxaline derivatives were confirmed by m.p, TLC and spectral data. All the synthesized compounds were screened for their antihistaminic activity. Compounds NVI-3, NVI-12, NVI-13, NVI-14 and NVI-15 were shown good \% protection of antihistamic activity.
\end{abstract}

Keywords 2,3-Diphenyl quinoxaline, Benzimidazole, Phenyl pyrazolo benzimidazolo quinoxaline, Antihistaminic activity.

\section{Introduction}

Benzimidazole moiety plays an important role in heterocyclic chemistry largely due to its wide range of biological activities ${ }^{1-4}$ such as antimicrobial, antitubercular, anti-inflammatory, anticancer etc. Quinoxaline derivatives have been reported to possess a wide variety of biological activities ${ }^{5-7}$. Notable among these are antioxidant, anti-inflammatory antimicrobial, anticancer and antihistamic activities. Drugs having pyrazoline ring system ${ }^{8-10}$ are well known for their anti-inflammatory, antioxidant, antihistamic, antimicrobial, antidepressant, hypoglycemic, hypotensive, anticarcenogenic activities etc. In view of the above facts, it was contemplated to design and synthesize some phenyl pyrazolo benzimidazolo quinoxaline derivatives (Scheme 1) by condensing benzimidazole quinoxaline chalcones with different aromatic acid hydrazides. All the synthesized compounds were screened for their antihistaminic activity. The structure of chalcones and phenyl pyrazolo benzimidazolo quinoxaline derivatives were confirmed by m.p, TLC, and spectral data. 
<smiles></smiles><smiles>c1ccc2[nH]cnc2c1</smiles>

( N II)<smiles></smiles>

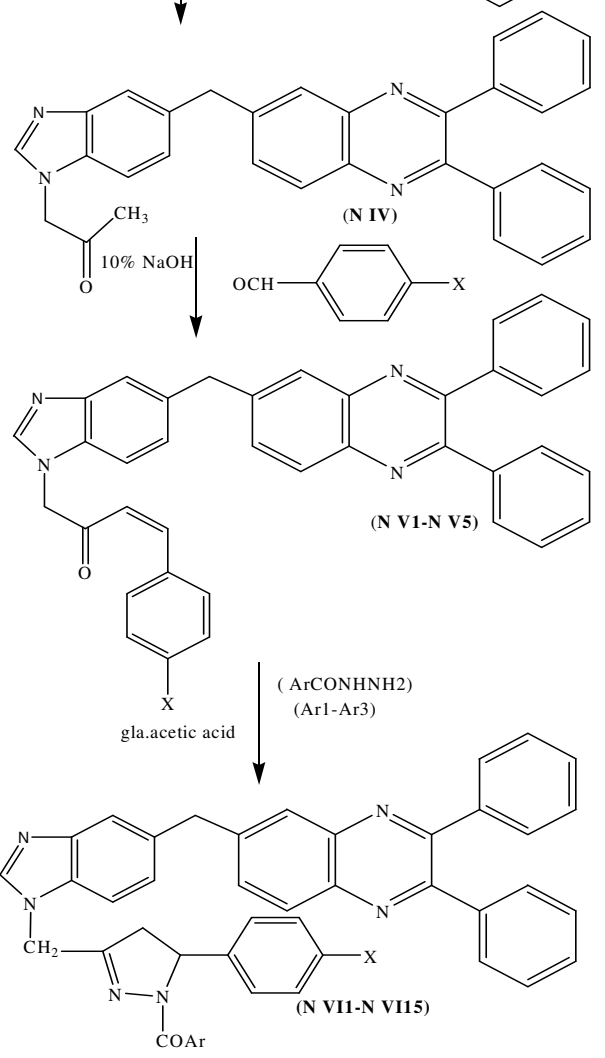

\section{Experimental}

Scheme 1

The melting point of the compounds were determined on a Thoshniwal electric melting point apparatus and the values were uncorrected. IR spectra of the compounds were recorded on a Thermo Nicolet Nexus670-FTIR, IICT, Hyderabad using KBr disc method. ${ }^{1} \mathrm{H}$ NMR spectra were recorded onAvance-300, IICT, Hyderabad using $\mathrm{CDCl}_{3}$ as solvent. Mass spectra were recorded on HITACHI RMU GL, IICT, Hyderabad. All the solvents used were of analytical grade. 
General procedure for synthesis of 6-((1H-benzo[d]imidazol-5-yl) methyl)-2, 3diphenylquinoxaline $\left(\right.$ NIII ${ }^{8}$

2,3 Diphenyl quinoxaline (NI) and benzimidazole (NII) were prepared following the literature method. NI and NII are linked with a methelyne bridge by treating equimolar quantities of NI and NII in suitable solvent with 35 parts formaldehyde solution and $35 \%$ $\mathrm{HCl}$, stirring for $4 \mathrm{~h}$. at $70{ }^{\circ} \mathrm{C}$ using magnetic stirrer. Solution was made alkaline using ammonia solution. Filtered the product and recrystallized with aqueous ethanol.

NIII: Yield 72\%, m.p $108{ }^{\circ} \mathrm{C}$, IR (KBr) in cm ${ }^{-1}: 1665$ (C=N str.), 1340 (C-N str.), 3085 (Ar-H str.) ${ }^{1} \mathrm{HNMR}\left(\mathrm{CDCl}_{3}\right) \delta: 5.0(\mathrm{~S}, 1 \mathrm{H}, \mathrm{N}-\mathrm{H}$ of benzimidazole $), 3.81(\mathrm{~S}, 2 \mathrm{H}$, methylene), 7.5-7.9 (m, 3H, quinoxaline), 7.2-7.4 (m, 10H, Ar-H), 7.0-8.1 (m, 3H, benzimidazole). Mass: $m / z$ : $428(\mathrm{M}+)$.

General procedure for Synthesis of 1-(5-((2,3-diphenylquinoxalin-6-yl)methyl)-1Hbenzo[d]imidazol-1-yl)propan-2-one $(\mathrm{NIV})^{9}$

A solution of NIII $(0.01 \mathrm{M})$ and chloroacetone $(0.01 \mathrm{M})$ were taken into $250 \mathrm{~mL}$ round bottom flask. Added to it $150 \mathrm{~mL}$ of dry acetone and $30 \mathrm{~g}$ of anhydride potassium carbonate and the reaction mixture were refluxed for $6 \mathrm{~h}$. below $75^{\circ} \mathrm{C}$. Filterate obtained was concentrated under vacuum and recrystallized with aqueous ethanol.

N IV: Yield 68\%, m.p $125^{\circ} \mathrm{C}$, IR (KBr) cm $\mathrm{cm}^{-1}: 1793$ (C=O str.), 1668 (C=N str.), 1340 (C-N str.), 3085 (Ar-H str.), 3323 (C-H str). ${ }^{1} \mathrm{HNMR}\left(\mathrm{CDCl}_{3}\right)$ 8: 2.0 (S, 3H, methyl), 3.8,4.8 (S, 4H, methylene), 7.4-7.9 (m, 3H, quinoxaline), 7.2-7.4 (m, 10H,Ar-H), 7.0-8.0 (m, $4 \mathrm{H}$, benzimidazole). Mass: $m / z$ : $468.2(\mathrm{M}+)$.

General procedure for synthesis of (Z)-4-phenyl-1-(5-((2,3-diphenylquinoxalin-6yl)methyl)-1H-benzo[d]imidazol-1-yl)but-3-en-2-one (NV1-NV5) $)^{10}$

Method of aldol condensation was followed. A solution of $\mathrm{NaOH} / \mathrm{KOH}(8 \mathrm{~mL}, 10 \%$ in water) was added drop wise to a well-stirred solution of NIV $(0.01 \mathrm{M})$ and $(0.01 \mathrm{M})$ of appropriate aldehyde in $20 \mathrm{~mL}$ ethanol. The reaction mixture was stirred for $24 \mathrm{~h}$. at cold conditions. Then diluted with ice water and acidified with con. HCl. Filtered the product and recrystallized with aqueous ethanol. The purity of the compound was checked by TLC and melting point.

NV 1: Yield: $73 \%$, m.p: $113{ }^{\circ} \mathrm{C}, \mathrm{IR}(\mathrm{KBr}) \mathrm{cm}^{-1}: 1773$ (C=O str.), 1668(C=N str.), 1340 (C-N str.), 3085 (Ar-H str.), 3323 (C-H str.) $\mathrm{cm}^{-1} .{ }^{1} \mathrm{HNMR}\left(\mathrm{CDCl}_{3}\right)$ 8: 3.8, 5.3 (S, 4H, methylene), 6.2,7.3(d, 2H,ethylene), 7.5-7.9(m, 3H, quinoxaline), 7.1-7.4 (m, 15H, Ar-H), 7.0-8.1 (m, 4H, benzimidazole). Mass: $m / z: 556.2(\mathrm{M}+)$

General procedure for Synthesis 6-((1-((1-benzyl-4, 5-dihydro-5-phenyl-1H-pyrazol-3-yl) methyl)-1H Benzo [d]imidazol-5-yl) methyl)-2, 3-diphenylquinoxaline (NVI1-NVI15) ${ }^{11}$

Chalcone NIV $(0.01 \mathrm{M})$ and aromatic acid hydrazide $(0.02 \mathrm{M})$ were taken in $20 \mathrm{~mL}$ glacial acetic acid and refluxed for $10 \mathrm{~h}$. above $130{ }^{\circ} \mathrm{C}$. The reaction mixture was concentrated and poured in $300 \mathrm{~mL}$ of ice-cold water and recrystallized with aqueous ethanol. The purity of the compound was checked by TLC and melting point.

NVI 1: Yield: $67 \%$, m.p: $121{ }^{\circ} \mathrm{C}, \mathrm{IR}(\mathrm{KBr}) \mathrm{cm}^{-1}: 1790$ (C=O str.), 1668 (C=N str.), 1339 (C-N str.), 3035 (Ar-H str.), 3320 (C-H str.) $\mathrm{cm}^{-1} .{ }^{1} \mathrm{HNMR}\left(\mathrm{CDCl}_{3}\right): 1.79,2.0$ (m, 2H, methylene), 3.8, $3.8(\mathrm{~S}, 4 \mathrm{H}$, methylene), $4.9(\mathrm{~m}, 1 \mathrm{H}$, methine), 7.5-7.9 (m, 3H, quinoxaline), 7.12-7.95 (m, 20H, Ar-H), 7.9-8.1 (m, 4H, benzimidazole). Mass: $m / z: 674.2(\mathrm{M}+$ ). 


\section{Pharmacological evaluation}

\section{Antihistaminic activity ${ }^{15}$}

\section{Histamine chamber method}

In this method, thirty two healthy adult guinea pigs of either sex divided into group of 2 animals each weighing around $400 \mathrm{~g}$, fasted overnight, were kept in histamine chamber, and exposed to histamine aerosol $(0.5 \%$ aqueous solution of histamine acid phosphate in a Nebulizer) until they collapse. Those that collapse within 2 minutes were revived with fresh air and used for this test. Twelve hours later, the animals were given an oral dose of test compound suspended in $1 \%$ acacia solution and after $1 \mathrm{~h}$ for absorption; the guinea pigs were again exposed to the same concentration of histamine aerosol. Those that do not collapse within 6 minutes are deemed protected. Percentage protection has been measured by using the following formula:

$$
\left[1-\mathrm{T}_{1} / \mathrm{T}_{2}\right] \times 100
$$

Where $\mathrm{T} 1$ was the mean of control preconvultion time in vehicle treated group and $\mathrm{T}_{2}$ was the mean of control preconvultion time in drug treated group. The results are shown in Table 2 .

Table 2. Antihistaminic studies of phenyl pyrazolo benzimidazolo quinoxaline derivatives.

\begin{tabular}{cccccc}
\hline Compd. & $\mathrm{X}$ & $\mathrm{Ar}$ & Mol. formula & $\begin{array}{c}\text { Onset of convulsions (s) } \\
\text { Mean } \pm \text { SD }\end{array}$ & \%Protection \\
\hline NVI 1 & $\mathrm{H}$ & $\mathrm{C}_{6} \mathrm{H}_{5}$ & $\mathrm{C}_{45} \mathrm{H}_{34} \mathrm{~N}_{6} \mathrm{O}$ & $990 \pm 90$ & 89.3 \\
NVI 2 & $\mathrm{OH}$ & $\mathrm{C}_{6} \mathrm{H}_{5}$ & $\mathrm{C}_{45} \mathrm{H}_{34} \mathrm{~N}_{6} \mathrm{O}_{2}$ & $1000 \pm 98$ & 89.5 \\
NVI 3 & $\mathrm{F}$ & $\mathrm{C}_{6} \mathrm{H}_{5}$ & $\mathrm{C}_{45} \mathrm{H}_{33} \mathrm{FN}_{6} \mathrm{O}$ & $1150 \pm 98$ & 90.8 \\
NVI 4 & $\mathrm{Cl}$ & $\mathrm{C}_{6} \mathrm{H}_{5}$ & $\mathrm{C}_{45} \mathrm{H}_{33} \mathrm{ClN}_{6} \mathrm{O}$ & $993 \pm 96$ & 89.4 \\
NVI 5 & $\mathrm{OCH}_{3}$ & $\mathrm{C}_{6} \mathrm{H}_{5}$ & $\mathrm{C}_{46} \mathrm{H}_{36} \mathrm{~N}_{6} \mathrm{O}_{2}$ & $999 \pm 91$ & 89.4 \\
NVI 6 & $\mathrm{H}$ & $\mathrm{OHC}_{6} \mathrm{H}_{4}$ & $\mathrm{C}_{45} \mathrm{H}_{34} \mathrm{~N}_{6} \mathrm{O}_{2}$ & $1000 \pm 92$ & 89.5 \\
NVI 7 & $\mathrm{OH}$ & $\mathrm{OHC}_{6} \mathrm{H}_{4}$ & $\mathrm{C}_{45} \mathrm{H}_{34} \mathrm{~N}_{6} \mathrm{O}_{3}$ & $1100 \pm 91$ & 90.4 \\
NVI 8 & $\mathrm{F}$ & $\mathrm{OHC}_{6} \mathrm{H}_{4}$ & $\mathrm{C}_{45} \mathrm{H}_{33} \mathrm{FN}_{6} \mathrm{O}_{2}$ & $1111 \pm 92$ & 90.5 \\
NVI 9 & $\mathrm{Cl}$ & $\mathrm{OHC}_{6} \mathrm{H}_{4}$ & $\mathrm{C}_{45} \mathrm{H}_{33} \mathrm{ClN}_{6} \mathrm{O}_{2}$ & $1020 \pm 92$ & 89.7 \\
NVI 10 & $\mathrm{OCH}$ & $\mathrm{OHC}_{6} \mathrm{H}_{4}$ & $\mathrm{C}_{45} \mathrm{H}_{36} \mathrm{~N}_{6} \mathrm{O}_{3}$ & $1022 \pm 96$ & 89.7 \\
NVI 11 & $\mathrm{H}$ & $\mathrm{ClC}_{6} \mathrm{H}_{4}$ & $\mathrm{C}_{45} \mathrm{H}_{33} \mathrm{ClN}_{6} \mathrm{O}$ & $1113 \pm 92$ & 90.5 \\
NVI 12 & $\mathrm{OH}$ & $\mathrm{ClC}_{6} \mathrm{H}_{4}$ & $\mathrm{C}_{45} \mathrm{H}_{33} \mathrm{ClN}_{6} \mathrm{O}_{2}$ & $1160 \pm 95$ & 90.9 \\
NVI 13 & $\mathrm{F}$ & $\mathrm{ClC}_{6} \mathrm{H}_{4}$ & $\mathrm{C}_{45} \mathrm{H}_{32} \mathrm{ClFN}_{6} \mathrm{O}$ & $1170 \pm 96$ & 91.0 \\
NVI 14 & $\mathrm{Cl}$ & $\mathrm{ClC}_{6} \mathrm{H}_{4}$ & $\mathrm{C}_{45} \mathrm{H}_{32} \mathrm{Cl}_{2} \mathrm{~N}_{6} \mathrm{O}$ & $1150 \pm 92$ & 90.8 \\
NVI 15 & $\mathrm{OCH}$ & $\mathrm{ClC}_{6} \mathrm{H}_{4}$ & $\mathrm{C}_{46} \mathrm{H}_{35} \mathrm{ClN}_{6} \mathrm{O}_{2}$ & $1180 \pm 92$ & 91.1 \\
Control & & & & $105 \pm 16$ & \\
CPM & & & & $1220 \pm 65$ & 91.3 \\
\hline
\end{tabular}

\section{Conclusions}

Synthesis of some phenyl pyrazolo benzimidazolo quinoxaline derivatives by condensing benzothiazolo quinoxaline chalcones with different aromatic acid hydrazides have been done successfully. The structure of chalcones and phenyl pyrazolo benzimidazole quinoxaline derivatives were confirmed by M.P, TLC and Spectral data. Physical data are shown in Table 1. All the synthesized compounds were screened for their Antihistaminic activity. Compounds NVI 3, NVI 12, NVI 13, NVI 14 and NVI 15 were shown good \% protection of antihistamic activity i.e., $90.8 \%, 90.9 \%, 91.0 \%, 90.8 \%$ and $91.1 \%$ respectively. 
Table 1. Physical data of phenyl pyrazolo benzimidazolo quinoxaline derivatives.

\begin{tabular}{lccclccc}
\hline Compd. & $\mathrm{X}$ & $\mathrm{Ar}$ & \multicolumn{1}{c}{ Mol. Formula } & Melting point range, ${ }^{\circ} \mathrm{C} \%$ Yield & $\mathrm{R}_{\mathrm{f}}$ value \\
\hline NVI1 & $\mathrm{H}$ & $\mathrm{C}_{6} \mathrm{H}_{5}$ & $\mathrm{C}_{45} \mathrm{H}_{34} \mathrm{~N}_{6} \mathrm{O}$ & $122-124$ & 70 & 0.8 \\
NVI2 & $\mathrm{OH}$ & $\mathrm{C}_{6} \mathrm{H}_{5}$ & $\mathrm{C}_{45} \mathrm{H}_{34} \mathrm{~N}_{6} \mathrm{O}_{2}$ & $114-115$ & 67 & 0.82 \\
NVI3 & $\mathrm{F}$ & $\mathrm{C}_{6} \mathrm{H}_{5}$ & $\mathrm{C}_{45} \mathrm{H}_{33} \mathrm{FN}_{6} \mathrm{O}$ & $112-115$ & 66 & 0.8 \\
NVI4 & $\mathrm{Cl}$ & $\mathrm{C}_{6} \mathrm{H}_{5}$ & $\mathrm{C}_{45} \mathrm{H}_{33} \mathrm{ClN}_{6} \mathrm{O}$ & $112-114$ & 78 & 0.91 \\
NVI5 & $\mathrm{OCH}_{3}$ & $\mathrm{C}_{6} \mathrm{H}_{5}$ & $\mathrm{C}_{46} \mathrm{H}_{36} \mathrm{~N}_{6} \mathrm{O}_{2}$ & $116-118$ & 67 & 0.8 \\
NVI6 & $\mathrm{H}$ & $\mathrm{OHC}_{6} \mathrm{H}_{4}$ & $\mathrm{C}_{45} \mathrm{H}_{34} \mathrm{~N}_{6} \mathrm{O}_{2}$ & $120-124$ & 66 & 0.81 \\
NVI7 & $\mathrm{OH}$ & $\mathrm{OHC}_{6} \mathrm{H}_{4}$ & $\mathrm{C}_{45} \mathrm{H}_{34} \mathrm{~N}_{6} \mathrm{O}_{3}$ & $120-124$ & 80 & 0.9 \\
NVI8 & $\mathrm{F}$ & $\mathrm{OHC}_{6} \mathrm{H}_{4}$ & $\mathrm{C}_{45} \mathrm{H}_{33} \mathrm{FN}_{6} \mathrm{O}_{2}$ & $108-110$ & 45 & 0.9 \\
NVI9 & $\mathrm{Cl}$ & $\mathrm{OHC}_{6} \mathrm{H}_{4}$ & $\mathrm{C}_{45} \mathrm{H}_{33} \mathrm{ClN}_{6} \mathrm{O}_{2}$ & $102-105$ & 45 & 0.8 \\
NVI10 & $\mathrm{OCH}_{3}$ & $\mathrm{OHC}_{6} \mathrm{H}_{4}$ & $\mathrm{C}_{45} \mathrm{H}_{36} \mathrm{~N}_{6} \mathrm{O}_{3}$ & $110-112$ & 67 & 0.83 \\
NVI11 & $\mathrm{H}$ & $\mathrm{ClC}_{6} \mathrm{H}_{4}$ & $\mathrm{C}_{45} \mathrm{H}_{33} \mathrm{ClN}_{6} \mathrm{O}$ & $120-122$ & 56 & 0.8 \\
NVI12 & $\mathrm{OH}$ & $\mathrm{ClC}_{6} \mathrm{H}_{4}$ & $\mathrm{C}_{45} \mathrm{H}_{33} \mathrm{ClN}_{6} \mathrm{O}_{2}$ & $120-122$ & 78 & 0.82 \\
NVI13 & $\mathrm{F}$ & $\mathrm{ClC}_{6} \mathrm{H}_{4}$ & $\mathrm{C}_{45} \mathrm{H}_{32} \mathrm{ClFN}_{6} \mathrm{O}$ & $131-133$ & 76 & 0.80 \\
NVI14 & $\mathrm{Cl}$ & $\mathrm{ClC}_{6} \mathrm{H}_{4}$ & $\mathrm{C}_{45} \mathrm{H}_{32} \mathrm{Cl}_{2} \mathrm{~N}_{6} \mathrm{O}$ & $130-134$ & 56 & 0.98 \\
NVI15 & $\mathrm{OCH}$ & $\mathrm{ClC}_{6} \mathrm{H}_{4}$ & $\mathrm{C}_{46} \mathrm{H}_{35} \mathrm{ClN}_{6} \mathrm{O}_{2}$ & $123-126$ & 54 & 0.81 \\
\hline
\end{tabular}

\section{Acknowledgements}

The authors are thankful to IICT Hyderabad for spectral analysis and also thankful to Geethanjali College of Pharmacy for providing facilities to carry out research work.

\section{References}

1. Joshi K C, Jain R, Dandia A and Sharma K, Indian J Chem., 1989, 28B, 698.

2. Marijana H, Kristina S, Piantanida I, Kralj M, Marjanovic M, Hasani M and Westman G, Eur J Med Chem., 2008, 43, 2877.

3. Mader M, Alfonso D, Chuan S, Rosanne B, Tiechao L, White W and Lopez U, Bioorg Med Chem Lett., 2008, 18, 179.

4. George D M, Larry D B, John M K, Timothy P B and Braulio S, Bioorg Med Chem Lett., 1997, 7, 2819.

5. Sandeep K and Devender shinda B, Bioorg Med Chem Lett., 2006, 16, 6181.

6. Dubey P K, Naidu A, Vijaya S and George Vineel B, Indian J Chem., 2005, 44B, 573.

7. Ganapathy $\mathrm{S}$, Ramalingam $\mathrm{P}$ and $\mathrm{Babu} \mathrm{Rao} \mathrm{CH}$, Indian J Heterocycl Chem., 2007, 16, 283.

8. Kumar A, Sharma S and Bajaj K, Indian J Chem., 2003, 42B(8), 1979.

9. Ragabasawaraj B and Sangapure S S, Indian J Heterocycl Chem., 2001, 11, 31.

10. Heas V, Roelof G and Cornelis A, Europ Pat Appl., 1981, 21, 506.

11. Suthakaran R, Nagarajan G, Balasubramaniam V, Suganthi K and Velrajan G, Indian J Heterocycl Chem., 2005, 14, 201.

12. Leonard J T, Yagnapriya S, Sridhar S K and Gunasekaran V, Indian J Heterocycl Chem., 2005, 14, 377.

13. Suthakaran R, Somasekhar G, Sridevi Ch, Mari kannan M, Suganthi K and Nagarajan G, Asian J Chem., 2007, 5, 3353.

14. Harinadha babu V, Sridevi Ch, Joseph A and Srinivasan K K, Indian J Pharm Sci., 2007, 66(8), 470.

15. Bhargava P N and Chaurasia M R, J Med Chem., 1968, 11, 908. 


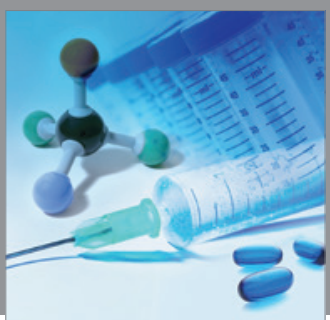

International Journal of

Medicinal Chemistry

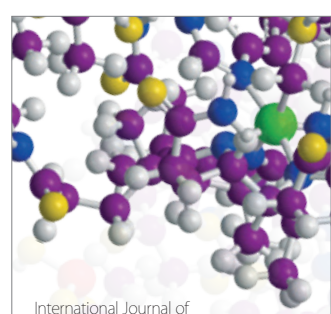

Carbohydrate Chemistry

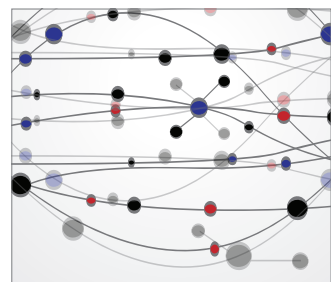

The Scientific World Journal
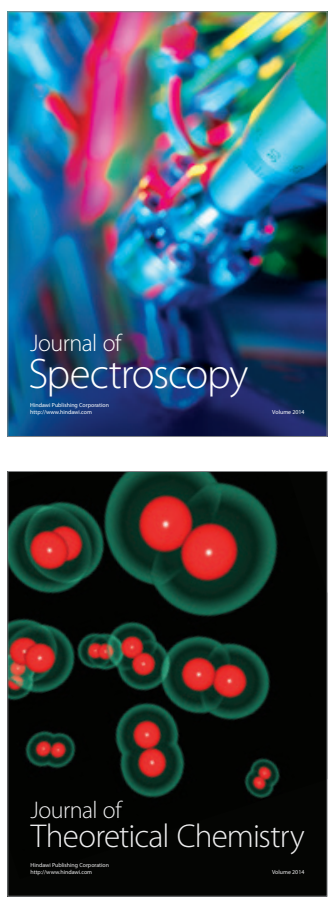
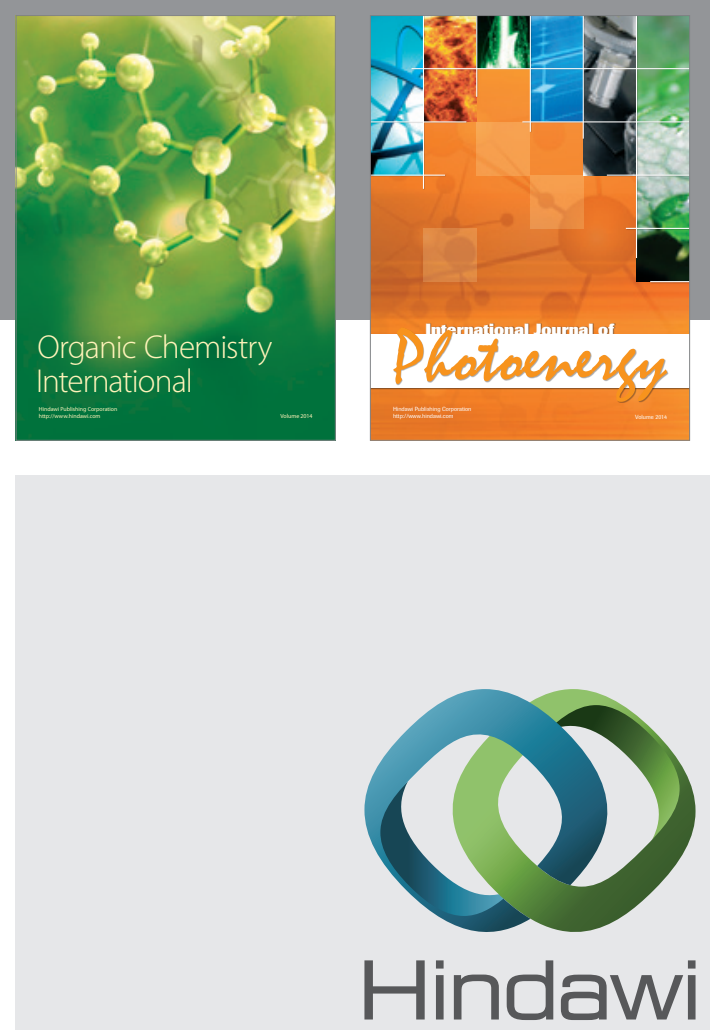

Submit your manuscripts at

http://www.hindawi.com
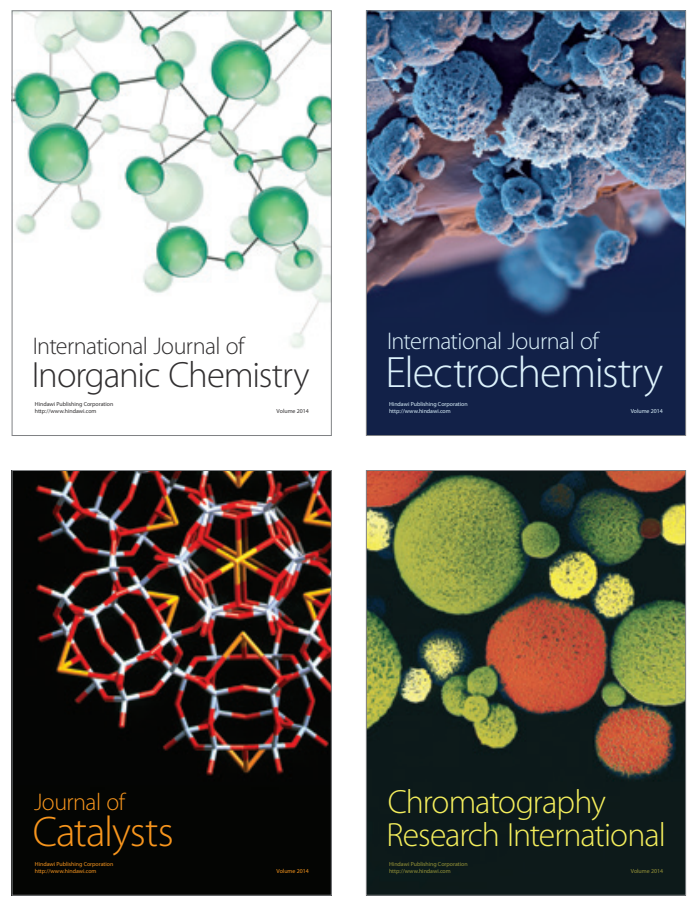
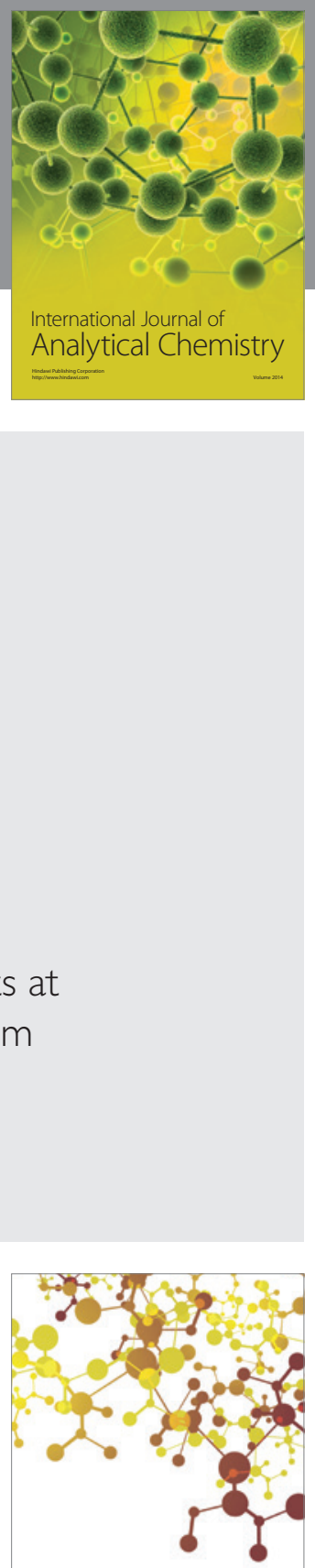

Journal of

Applied Chemistry
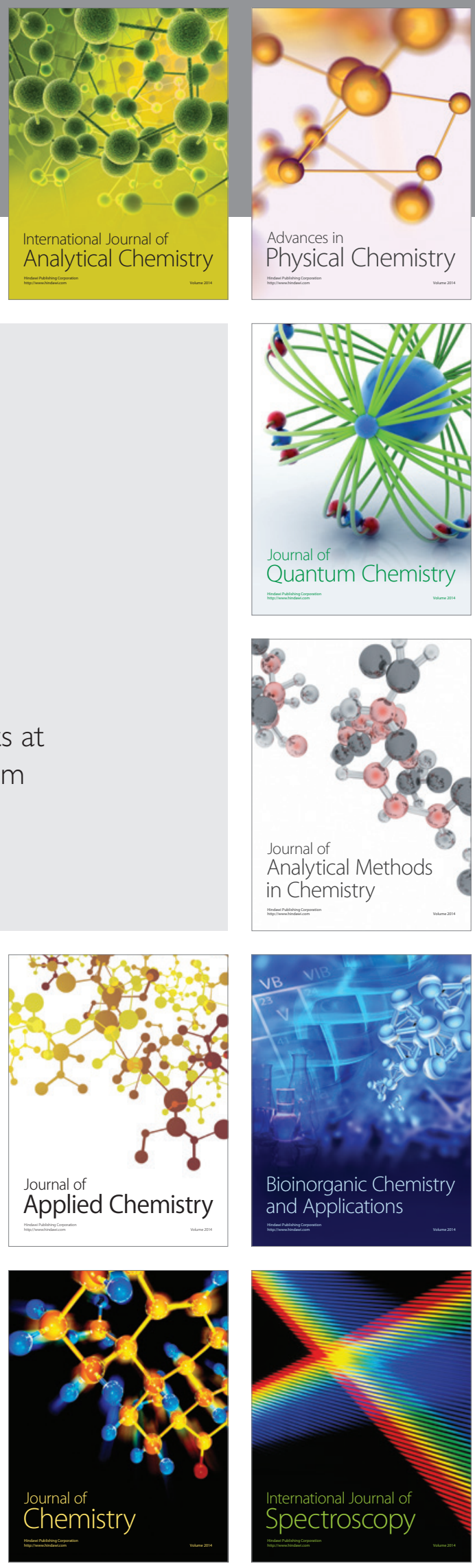